\title{
Micrometric particle's isotopics: An ultra-sensitive tool to detect nuclear plant discharge in the environment
}

\author{
S. Baude, M.C. Lartière, O. Marie and R. Chiappini \\ CEA, Service Radioanalyses, Surveillance, Environnement, 91680 Bruyères-te-Châtel, France
}

\begin{abstract}
To monitor the impact of a nuclear plant on the environment, common analytical methods consist of measuring the entire sample giving an average value for the element or for the isotopic ratios of this element for the "bulk " sample content. For uranium and plutonium, quantification or isotopic ratio measurements are routinely performed in bulk analysis with mass spectrometry (ICPMS or TIMS), with sensitive limits of detection.

To assess a better understanding of pollution sources and to get more precise information from environmental samples, new methods based on single particle measurements of $U$ and $P u$ isotopics were developed.

We discuss the clean-room based process that gives isotopic signatures for micron-scaled grains released in the environnent by nuclear facilities. It decreases the total sample size needed to be measured in common analytical methods, and allows us to eliminate naturally occurring uranium.
\end{abstract}

\section{INTRODUCTION}

Airborne particle discharge is one of the main concerns for industrial plants.

Some common analytical methods (TIMS: thermal ionization mass spectrometry, ICPMS: inductively coupled plasma mass spectrometry and associated isotope dilution methods) can provide bulk content assessment in environmental samples from nuclear plant using fissile material.

Therefore, pictogram amounts of material give precise isotope ratios and the limits of detection for high resolution ICP mass spectrometer are in the very low femtogram range for $\mathrm{Pu}$.

An ultra-sensitive detection for particles is needed to discriminate the tiny amounts of material released by nuclear facilities from natural background and global fallout.

Moreover, these airborne discharges are generally composed of multiple sources of emission.

That's the reason why a new methodology has been developed to isolate single grains within a sample and run individual particle isotopic measurements.

This technique gives more sensitivity leading to a better understanding of pollution sources and more precise information is collected from environmental samples.

Then, international safeguards verifications and non-proliferation issues can be addresses by the measurement of particles collected on samples like cotton swipes, air sampling filters or foliage material.

\section{BASIC PRINCIPLES FOR FISSION TRACK/TIMS TECHNIQUE}

The basic principles for particle analysis using the so-called fission tracks TIMS technique are as follows:

The sample is checked for non-contamination by High Resolution Gamma Spectrometry and then processed in a class 10 clean laboratories.

After sample preparation, neutron irradiation is performed to locate $U$ or Pu containing particles by locating their fission tracks. Then, after a micro-manipulation step under an optical microscope, thermal ionization mass spectrometric analysis is run using a sensitive laboratory built pulse counter. 


\subsection{Sample preparation}

As mentioned before, the sample preparation takes place in class 10 clean laboratories.

Particles are removed from a swipe or from a filter by ultra-soneration or sometime by the mean of a low temperature plasma asher. We add collodion to the suspension in ethanol and spread all the particles on 30 millimeters polycarbonate disks (Lexan).

Once dried, each disk is covered with a solid state nuclear track detector made of another thin Lexan disk. 30 disks per sample are mounted in a polypropylene rabbit tube and sent to Saclay (CEA center, south from Paris) for irradiation on the Orphée research reactor. A neutron flux of $1.3 \times 10^{13} \mathrm{~cm}^{-2} \cdot \mathrm{s}^{-}$ ${ }^{1}$ is applied for $1 \mathrm{~min}$ and allows us to identify particles that contain fissile atoms from their fission tracks revealed after chemical etching of the detector (Figure 1).

\subsection{Tracking down particles containing fissile atoms}

To track down and locate particles containing fissile atoms, the two Lexan disks are placed in a side-byside assembly on the microscope stage (Figure 2).

Marks recorded before irradiation help shift the stage from a given group of fission tracks on the etching disk, to the location of the particle that gave these fissions, on the other slide.

To select particles for analysis, we select the biggest fission track stars, which correspond to the largest amount of ${ }^{235} \mathrm{U}$ or ${ }^{239} \mathrm{Pu}$. It can be either the biggest particle or a high enrichment in these isotopes. It is important to remember that the 100 or 200 hundred micrometer stars we can see on Figure I Lexan disk come from 1 or 2 micrometer particles which contain a few picograms of $\mathrm{U}$ or $\mathrm{Pu}$.

The particles are collected by micro-manipulation under a reverse optical microscope. A carbide needle with a tip of 2-5 micrometers in diameter is used to cut a squared peace of collodion: 40-50 micrometers (Figure 3).

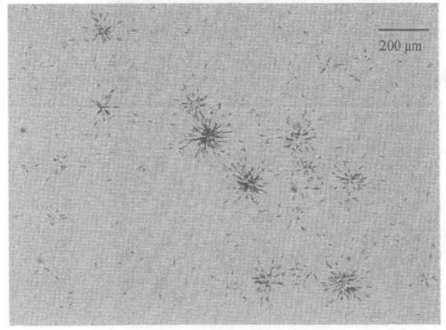

Figure 1. Fission tracks for uranium particles In Lexan after $1 \mathrm{~min}$ irradiation.

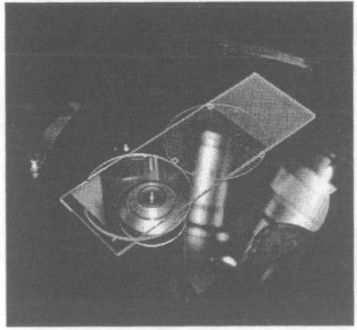

Figure 2. Side by side assembly to Relocate particles from their fission tracks. 


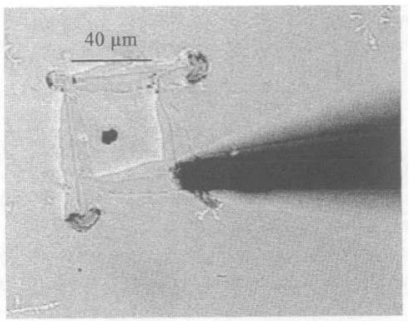

Figure 3. Micromanipulation using a carbide needle under reverse optical microscope.

The peace of collodion is deposited with sucrose onto a boat-shaped high purity rhenium filament used for TIMS isotope ratio measurement.

\section{APPLICATION TO THE DETECTION OF URANIUM ENRICHMENT}

\subsection{Analytical capabilities}

Here, we will give two examples where emission sources can be identified by particle analysis.

The plot shown in Figure 4 represents the number of particles measured for a given range of enrichment. Individual $235 / 238$ ratios are reported in Table 1 with uncertainties.

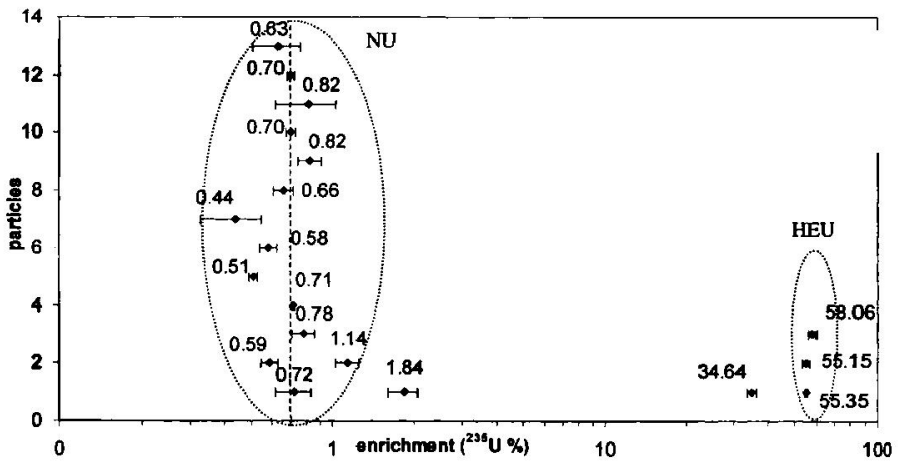

Figure 4. Identification of discharge sources in a made-up sample ${ }^{\prime}$. Particles are plotted with one standard deviation in groups corresponding to natural enrichment-like and high enrichment-like.

In this made-up sample (from IAEA'), 15 particles were found to have 235 close to natural uranium that would probably reveal natural uraniurn handling. And for this sample, we can see that only 3 or 4 particles give isotopic signature of a process involving highly enriched uranium.

\footnotetext{
'This sample is a quality control sample provided in the IAEA NWAL (Network of Analytical Laboratories)
} 
In a similar case, the bulk measurement would have revealed a false picture of the discharge in the environment and the average isotopic ratio wouldn't have allowed us to identify the different sources of emission.

Table 1 . Individual ratios for uranium particles plotted on Figure 4 graph.

\begin{tabular}{|c|c|c|c|c|c|c|c|c|c|}
\hline Particle & P1-89 & P1-86 & P1-70 & P3-64 & P1-90 & P1-66 & P2-66 & P1-63 & P1-69 \\
\hline${ }^{233} U / /^{236} U$ & 0.0187 & 0.0071 & 0.0073 & 0.0079 & 0.0064 & 0.0059 & 1.2398 & 1.3841 & 0.0051 \\
\hline Std. Dev, & 0.0022 & 0.0003 & 0.0011 & 0.0008 & 0.0013 & 0.0004 & 0.0087 & 0.0415 & 0.0002 \\
\hline pa & -87 & P3-63. & P2-86 & P1-82 & P1-64 & P2-63 & $\mathbf{P 2 - 8 7}$ & P3-87 & P2-60 \\
\hline${ }^{235} \mathrm{U} / \mathrm{J}^{236} \mathrm{U}$ & 0.0071 & 0.0115 & 0.0083 & 0.5301 & 1.2296 & 0.0059 & 0.0044 & 0.0066 & 0.0072 \\
\hline Std. Dev. & 0.0002 & 0.0012 & 0.0021 & 0.0212 & 0.0369 & 0.0004 & 0.0011 & 0.0006 & 0.0001 \\
\hline
\end{tabular}



Figure 5. Particles from an auxiliary area plotted according to their 235 enrichment. Errors bars represent one standard deviation.

\subsection{Example from the uranium enrichment cycle}

The second example we will deal with comes from the uranium enrichment cycle. The plant handle uranium from natural to high enriched levels. The swipe was sampled from an auxiliary area.

Once again, particles are plotted with regards to their 235 enrichment in Figure 5 . We can see that with less than 20 particles analyzed, natural, and low enriched and high enriched uranium can be detected due to the great sensitivity of fission tracks.

\section{COMPLEMENTARY TECHNIQUES}

The very high sensitivity to enrichment in ${ }^{235} \mathrm{U}$ or ${ }^{239} \mathrm{Pu}$ obtained with the combined fission track and TIMS techniques has to be balanced with the long time of analysis due to a minute process involving a nuclear reactor. Thus, future developments for particle analysis include search for faster screening techniques.

If laser ablation coupled with ICPMS seems to be difficult to set up, $\mathrm{X}$ ray micro-analysis and secondary ion mass spectrometry are more promising complementary tools. We will just introduce their capabilities through the very first tests we performed. 


\subsection{SEM/EDX capabilities for particles}

With energy dispersive $\mathrm{X}$ ray micro-analysis, $\mathrm{U}$ and $\mathrm{Pu}$ particles can be identified directly in the non conductive collodion layer if we use a low vacuum electron microscope.



Figure 6. Uranium oxide particle of a few micrometers identified by the EDX spectrum shown here.

We can also use an automatic search routine, similar to gun shot residue analysis in order to screen the sample for $\mathrm{U}$ and $\mathrm{Pu}$ richness. This method also opens doors for further studies with biological effects of particles including radionuclides.

\subsection{SIMS (Secondary Ion Mass Spectrometry)}

Secondary Ion mass spectrometry can be used for particle analysis in two different approaches [1]. First, image of ion signais like uranium 238 picture on Figure 6 can be obtained to locate particles. A microprobe mode can then provide a good isotope ratio measurement for each single grain.

On the diagram in Figure 8, we compare the sensitivity to select enriched uranium of the SIMS measurement to the fission track TIMS method. From a made up sample

The results indicate that for 20 particles analyzed by each method, the high sensitivity of fission tracks allows us to detect two classes of enriched uranium, where the SIMS measurements' only suggest one.

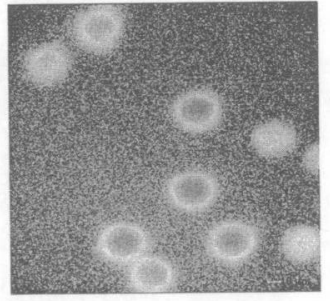

Figure 7.238 uranium ion image for $1-1.5 \mu \mathrm{m}$ particles.

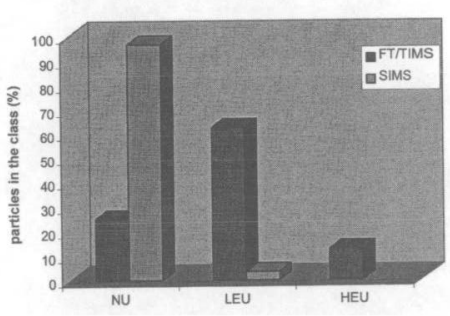

Figure 8. SIMS and FT/TIMS methods for a made-up sample?

\footnotetext{
'Measurements were done on a CEA / ENSMP (Ecole Nationale Supérieure des Mines de Paris) shared Cameca IMS of instrument. Thanks to MM. Louis Raimbault (ENSMP) and Gabriele Tamborini (ITU) for usefull help.

${ }^{2}$ Courtesy of M. Gabriele Tamborini, Institute for Transuranium Elements, European Commission JRC.

${ }^{3}$ This is another a quality control sample provided in the IAEA NWAL (Network of Analytical Laboratories).
} 


\section{CONCLUSION}

Isotopic signatures on micron-scaled grains can be identified by different methods. Techniques based on fission tracks provide a very high sensitivity to detect the higher enrichment but suffer from time consuming steps in sample processing. Complementary methods are available and need to be investigated in terms of sensitivity to provide a fast screening mode. More information could then be obtained for each particle, including chemical composition.

\section{References}

[1] TAMBORINI, G., et al., "Application of secondary ion mass spectrometry to the identification of single particles of uranium and their isotopic measurement", Spectrochemica Acta B, 53 (1998) 1289 1302 